CERN-PH-TH/2005-186, ACT-10-05, MIFP-05-26

October 2005

\title{
Robust Limits on Lorentz Violation from Gamma-Ray Bursts
}

\author{
John Ellis ${ }^{a}$, N.E. Mavromatos ${ }^{b}$, D.V. Nanopoulos ${ }^{c}$, \\ A.S. Sakharov ${ }^{a, d}$ and E.K.G. Sarkisyan ${ }^{e}$
}

\begin{abstract}
We constrain the possibility of a non-trivial refractive index in free space corresponding to an energy-dependent velocity of light: $c(E) \simeq c_{0}(1-E / M)$, where $M$ is a mass scale that might represent effect of quantum-gravitational space-time foam, using the arrival times of sharp features observed in the intensities of radiation with different energies from a large sample of gamma-ray bursters (GRBs) with known redshifts. We use wavelet techniques to identify genuine features, which we confirm in simulations with artificial added noise. Using the weighted averages of the time-lags calculated using correlated features in all the GRB light curves, we find a systematic tendency for more energetic photons to arrive earlier. However, there is a very strong correlation between the parameters characterizing an intrinsic time-lag at the source and a distance-dependent propagation effect. Moreover, the significance of the earlier arrival times is less evident for a subsample of more robust spectral structures. Allowing for intrinsic stochastic time-lags in these features, we establish a statistically robust lower limit: $M>0.9 \times 10^{16} \mathrm{GeV}$ on the scale of violation of Lorentz invariance.
\end{abstract}

CERN-PH-TH/2005-186

October 2005

a Theory Division, Physics Department, CERN, 1211 Geneva 23, Switzerland

${ }^{b}$ Theoretical Physics, Department of Physics, King's College London, Strand, London WC2R 2LS, UK

${ }^{c}$ Department of Physics, Texas A \& M University, College Station, TX 77843, USA;

Astroparticle Physics Group, Houston Advanced Research Center (HARC), Mitchell Campus, Woodlands, TX 77381, USA;

Academy of Athens, Division of Natural Sciences, 28 Panepistimiou Avenue, Athens 10679, Greece

${ }^{d}$ Swiss Institute of Technology, ETH-Zürich, 8093 Zürich, Switzerland

e EP Division, Physics Department, CERN, 1211 Geneva 23, Switzerland;

Department of Physics, the University of Manchester, Manchester M13 9PL, UK 


\section{Introduction}

The construction of a quantum theory of gravity remains an elusive goal [1], even more so the formulation of incisive experimental tests of such a theory. There is a general expectation that quantum fluctuations in the background space-time metric would make it appear 'foamy' on short time and distance scales [2], for which models have been proposed in various frameworks such as non-critical string theory [3], loop quantum gravity [4], string theory [5], double special relativity [6] and an effective field theory approach [7]. It has been suggested that the propagation of light through this space-time foam might exhibit a non-trivial dispersion relation in vacuo [8], corresponding to Lorentz violation via an energy-dependent velocity of light. It was also pointed out that one powerful way to probe this possibility may be provided by distant astrophysical sources of energetic photons that exhibit significant and rapid variations in time, such as gamma-ray bursters (GRBs) [8]. The possibility of an energy-dependent velocity of light: $c(E) \simeq c_{0}(1-E / M)$, where $c_{0}$ is a limiting low-energy velocity of light and $M$ is a mass scale that might represent effect of quantum-gravitational space-time foam, has subsequently been explored and constrained by many phenomenological analyses using pulsars and active galactic nuclei (AGNs) as well as GRBs. Various limits in the range $M>10^{15}$ to $10^{17} \mathrm{GeV}$ have been reported [9, 10, 11, 12, 13]. The importance of Lorentz violation for high-energy cosmic rays has also been considered [14], and laboratory probes of different forms of Lorentz violation have also been investigated [15].

In order to identify an effect as radical as Lorentz violation, one must minimize the uncertainties by a careful statistical analysis and control of possible systematic errors. One cannot rely on a single source, for which it would be impossible to distinguish an intrinsic time-lag at the origin from a delay induced by propagation in vacuo, particularly if the observation of the source is uncertain in a crucial energy band. For this reason, we have pioneered the systematic analysis [16] of statistical samples of GRBs at a range of different redshifts, and we have introduced techniques from signal processing such as wavelet analysis to identify and correlate genuine features in the intensities observed in different energy bands. This technique has the advantage that it can extract time-dependent features from the signals of many GRBs, even weak ones, comparing measurements with more than one spectral channel. Our original analysis [16] used data from the BATSE and OSSE instruments on the CGRO satellite [17]. Recently, several new instruments such as HETE [18] and SWIFT [19] have provided additional, larger samples of GRBs with known redshifts.

In this paper, we combine these newer data with the older BATSE data to establish a hard limit on the foamy mass scale $M$. We supplement the wavelet technique used earlier with a statistical noise technique for identifying genuine features in the light curves. We also make a careful treatment of systematic uncertainties associated with the possibility of intrinsic time-lags in the sources and treat the data with standard tools for evaluating the statistical significance of possible signals. A two-parameter fit to $M$ and a fixed time-lag would seem to suggest a significant foamy effect. However, the two parameters are highly correlated and the quality of the fit is very poor, suggesting the existence of some additional 
source of error and/or uncertainty. If one does not know why a fit is poor, the Particle Data Group suggests rescaling the estimated errors so that the overall $\chi^{2} /$ d.o.f. $\simeq 1$. Applying this procedure to the full data set of time-lags in features found in a large sample of GRBs, we are left with a priori $4 \sigma$ evidence that higher-energy photons tend to arrive earlier than lower-energy photons. Selecting the most robust spectral features reduces the significance of the time-delay effect to the $2 \sigma$ level when we rescale the errors in this way. However, one natural hypothesis for the origin for the poorness of the fit is that the intrinsic time-lags at the sources are distributed stochastically. Including this possibility in an analysis of the full data set and choosing the r.m.s. spread of the intrinsic time-lags so that the the overall $\chi^{2} /$ d.o.f. $\simeq 1$, we find that the significance of any propagation effect is reduced to the $1 \sigma$ level.

Using the robust subsample and either rescaling the errors or allowing for stochastic intrinsic time-lags, we find rather similar lower limits on the quantum-gravity scale $M$, which are similar whether we estimate them within a a Bayesian approach or use the likelihood function. Taking the smallest of the numerical limits on $M$, we find onservatively $M>0.9 \times 10^{16} \mathrm{GeV}$ at the $95 \%$ confidence level. There is no stronger robust limit on such a form of Lorentz violation.

\section{Time Delays induced by Lorentz Violation in the Expanding Universe}

To search for possible effects of violation of Lorentz invariance, we compare the propagation of photons with energies much smaller than the mass scale $M$ characterizing the difference of the vacuum refractive index from unity ${ }^{1}$. A small difference between the velocities of two photons with an energy difference $\Delta E$, emitted simultaneously by a remote cosmological source, would lead to a time-lag between the arrival times of the photons. Taking into account that the effect on the propagation of photons due to the expansion of the Universe, one has [16] the following dependence on redshift $z$ of the induced differences in the arrival times of the two photons with energy difference $\Delta E$ :

$$
\Delta t_{\mathrm{LV}}=H_{0}^{-1} \frac{\Delta E}{M} \int_{0}^{z} \frac{d z}{h(z)},
$$

where $H_{0}$ is the Hubble expansion rate and

$$
h(z)=\sqrt{\Omega_{\Lambda}+\Omega_{M}(1+z)^{3}} .
$$

Throughout this paper, we assume a spatially-flat Universe: $\Omega_{\text {total }}=\Omega_{\Lambda}+\Omega_{M}=1$ with cosmological constant $\Omega_{\Lambda} \simeq 0.7$ : see [21] and references therein.

\footnotetext{
${ }^{1}$ In four-dimensional models of quantum gravity this scale may be of the same order as the Planck mass $M_{P}$, but it might be much smaller in some models with large extra dimensions [20].
} 
To look for such a vacuum refractive index effect, we need a distant, transient source of photons of different energies, preferably as high as possible. One may then measure the differences in the arrival times of sharp transitions in the signals in different energy bands. GRBs are at cosmological distances, as inferred from their redshifts, and exhibit many transient features in their time series in different energy bands. In comparison, the observed active galactic nuclei (AGNs) have lower redshifts and broader time structures in their emissions, but have the advantage of higher photon energies. Observable pulsars [12] have very well-defined time structures in their emissions, but are only at galactic distances.

The key issue in all such probes is to distinguish the effects of the possible violation of Lorenz invariance from any intrinsic delay in the emission of photons of different energies by the source. It is obvious from Eq. (1) that any effect of violation of Lorentz invariance should increase with the redshift of the source, whereas source effects would be independent of the redshift in the absence of any cosmological evolution effects [22]. Therefore, in order to disentangle source and propagation effects, it is preferable to use transient sources with a broad spread in known redshifts $z$. Thus, one of the most model-independent ways to probe the time-lags that might arise from quantum gravity is to use the GRBs with known redshifts, which range up to $z \sim 6$.

In the present paper, we exploit a sample of 35 GRBs with known redshifts, including 9 GRBs detected by the Burst And Transient Source Experiment (BATSE) aboard the Compton Gamma Ray Observatory (CGRO), 15 detected by the High Energy Transient Explorer (HETE) satellite and 11 detected by the SWIFT satellite. We used public archives for the light curves obtained with BATSE [17] HETE [18] and SWIFT [19]. The information on the redshifts has been collected from [23]. The GRBs in our sample are listed in Table 1 together with their redshifts [24-45] and the time-lags we extract from their light curves. The BATSE light curves were recorded with a time resolution of $64 \mathrm{~ms}$ in four spectral channels with boundaries at approximately 25, 55, 115 and $320 \mathrm{KeV}$. The HETE time resolution is rather coarser, namely $164 \mathrm{~ms}$ in three energy bands in a similar energy range. The light curves of SWIFT are also produced with a resolution of $64 \mathrm{~ms}$ in four energy ranges that are almost identical those of BATSE. In this analysis, we look for spectral time-lags in the light curves recorded in the $115-320 \mathrm{KeV}$ energy band relative to those in the lowest $25-55 \mathrm{KeV}$ energy band, thereby maximizing the lever arm in photon energies provided by the available data. We renormalize the time-lags obtained from the HETE data with respect to the two above-mentioned BATSE energy bands, by a factor corresponding to the energy dependence in (1) and determined by the ratio of the energy differences $\Delta E$ between the boundaries of the HETE and BATSE spectral channels. We also renormalized some of the SWIFT data, because we used more closely-spaced energy bands for some of the SWIFT GRBs. 


\section{Detection of Genuine Time-Dependent Features in GRB Light Curves}

We wish to study a possible redshift-dependent time offset between different energy bands, and so constrain any possible difference in the propagation speeds of photons of different energies. In order to measure the times of flight of photons, we need to identify features in the GRB light curves where the emission rates change very rapidly. The first step is to introduce criteria specifying the points in the GRB light curves where the most significant variations take place, and characterize the degree of variability at each such point. Here we follow the approach of [16], using the Lipschitz exponents $\alpha_{L}$ to identify the variation points of the light curves measured in different energy bands. The measurability of the Lipschitz exponents provides a quantitative criterion for identifying genuine variation points belonging to different energy bands, which may then be correlated. Specifically, for this analysis we identify and use the most singular, so-called 'genuine' variation points, at which the Lipschitz exponents are smaller than 1 (for details, see [16]).

Following the technique developed in [16], we first use the wavelet shrinkage procedure [46] based on the thresholding of the discrete wavelet transform (DWT). This consists of using using the DWT transform to break the intensity profile down into successive approximations on different time scales, starting with a relatively coarse approximation and progressing to successive levels of detail on finer and finer scales. We then remove the wavelet coefficients below a specified threshold value, and diminish the others by the value of this threshold value. We then reconstruct the intensity profile by the inverse DWT, using the thresholded wavelet coefficients. This denoising procedure allows us to separate the structure of the signal from the noise, while retaining information about the position of true time structures in the signal. Subsequently, we apply the continuous wavelet transform (CWT) 'zoom' technique to the denoised intensity profiles, which identifies the times of genuine variation points and estimates their Lipschitz exponents in each spectral band we utilize. Genuine variational structures found at similar times in different spectral bands are considered to be manifestations of one and the same process at the source, if the values of their Lipschitz exponents are similar to each other. We identify such variation points in pairs. Since variation points with $\alpha_{L}$ substantially exceeding 1 exhibit only smooth transitions of the signal, and hence do not mark sharp dynamical changes at the source and are more likely to be spurious, we discard them from the subsequent analysis. The light curves of most of the GRBs in our dataset exhibit more than one genuine variation point with $\alpha_{L}<1$. The differences between the times of the genuine Lipschitz variation points in the higher-energy spectral band and their counterparts in the lower-energy spectral band are taken as measures of the possible time delay.

There are several sources of uncertainties in the analysis outlined above, which may arise from both our procedure for estimating the DWT intensity profiles and the CWT 'zoom' technique for detecting genuine variation points. Going beyond [16], here we check the statistical stability of the procedure used to determine the positions of the genuine variation points by making simulations in which a Gaussian distributed noise was added 
to the original light curves recorded by the instruments, in each energy band. The amount of the artificial noise was chosen to be consistent with the thresholding signal-to-noise ratio (SNR) revealed after the DWT, and with the power of the original signal measured in each time bin. Following each iteration of the artificial noise, the DWT thresholding procedure described above [16] and the CWT 'zoom' technique were performed repeatedly, until the values of the amplitudes at the genuine variation points obtained in each iteration and values of their Lipshitz exponents approached normal distributions. To achieve reasonable convergence of the distributions of the above-mentioned quantities to normal distributions, we had typically to make 1000 to 2000 artificial realizations per energy band. This procedure of contaminating the real data with artificial noise allows us to accumulate statistics sufficient to estimate the errors in the determination of the positions of the genuine variation points [47]. The identifications of pairs of genuine variation points belonging to different spectral bands but associated with the same emission process at the source are then made by looking for equality of the mean values of the Lipshitz exponents within the standard errors of the set of values obtained in the simulations ${ }^{2}$.

\section{Time-lags in Emissions from GRBs and the Limit on the Violation of Lorentz Invariance}

We now discuss our procedure for analyzing the possible existence of a Lorentz-violating contribution (1) to the observed time-lags. As discussed already in Section 2, this may be accompanied by a priori unknown intrinsic energy-dependent time-lags caused by unknown poperties of the sources. We take this possibility into account by fitting the measured timelags with the inclusion of a term $b_{\mathrm{sf}}$ specified in the rest frame of the source. Therefore, the resulting observed arrival time delays are fitted by two contributions:

$$
\Delta t_{\mathrm{obs}}=\Delta t_{\mathrm{LV}}+b_{\mathrm{sf}}(1+z)
$$

reflecting the possible effects of Lorentz violation and intrinsic source effects, respectively. Rescaling (3) by a factor $(1+z)$, we arrive at a simple linear fitting function

$$
\frac{\Delta t_{\mathrm{obs}}}{1+z}=a_{\mathrm{LV}} K+b_{\mathrm{sf}}
$$

where

$$
K \equiv \frac{1}{1+z} \int_{0}^{z} \frac{d z}{h(z)}
$$

is a non-linear function of the redshift which is related to the measure of cosmic distance in (1). The coefficient $a_{\mathrm{LV}}=H_{0}^{-1} \frac{\Delta E}{M}$ of the slope in $K$ is connected to the scale of

\footnotetext{
${ }^{2}$ This procedure also enables us to tag the most robust time structures in the light curves, which we discuss later.
} 

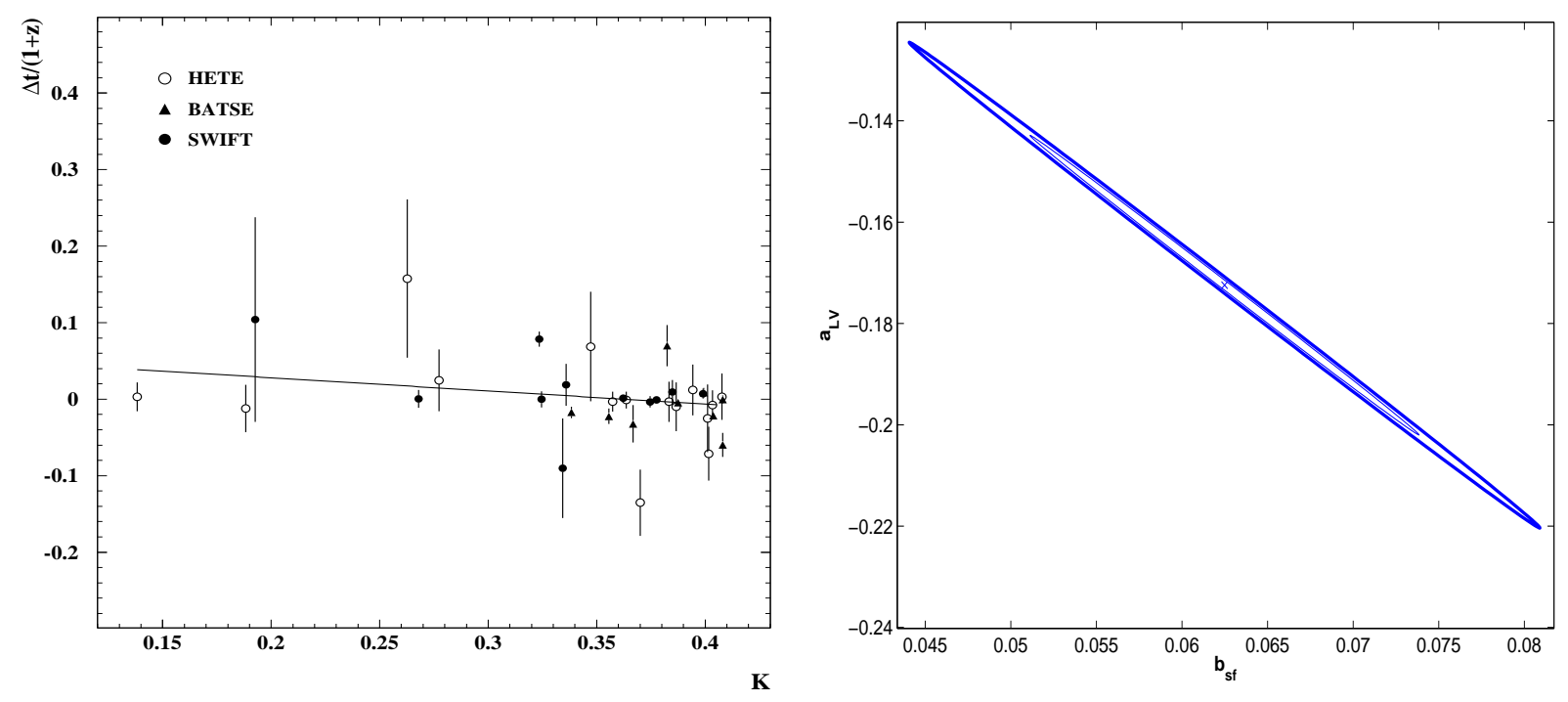

Figure 1: Left panel: The rescaled spectral time-lags between the arrival times of pairs of genuine sharp features detected in the light curves of the full set of 35 GRBs with measured redshifts observed by BATSE (9 light curves with a time resolution of $64 \mathrm{~ms}$ ), HETE (15 light curves with a time resolution of $164 \mathrm{~ms}$ ) and SWIFT (11 light curves with a time resolution of $64 \mathrm{~ms}$ ) shown in Table 1 normalized to the difference between the energies of the third and first BATSE spectral bands. The errors in the redshifts and hence in $K$ are negligible: the errors in the time-lags are estimated by the wavelet analysis described in the text. Also shown in the left panel is a linear fit to these data with $\chi^{2} /$ d.o.f. $=148.2 / 33$. Right panel: The error ellipse in the slope-intercept plane for the fit (6). The 68\% and 95\% confidence-level contours are represented by the inner and outer lines, respectively.

Lorentz violation, whereas the intercept $b_{\text {sf }}$ represents the possible unknown intrinsic timelag inherited from the sources. Initially, we assume this to be universal in the rest frame of every GRB, but later we investigate the possibility that this varies stochastically.

In order to probe the energy dependence of the velocity of light that might be induced by quantum gravity, we compile the available data as functions of the variable $K(5)$. Since (4) exhibits a linear dependence on $K$, we perform a linear regression analysis of the rescaled time-lags (1) between the genuine variation points in the higher-energy bands and their Lipschtz counterparts in the lowest-energy bands of the instruments. The result of a straight-line fit (4) to the rescaled time-lags extracted from the 35 light curves listed in Table 1, which were collected by the BATSE, HETE and SWIFT instruments, is shown in the left panel of Fig. $1^{3}$.

Although the data points exhibit some scatter, the majority of the time-lags extracted from the BATSE instrument agree within errors with the cross-correlation analysis of [48].

\footnotetext{
${ }^{3}$ As already mentioned, since the data from the HETE and SWIFT instruments are made available in slightly different energy bands, we have rescaled the time-lags and errors from Table 1 by the ratios of the energy differences of the HETE and SWIFT data relative to those of the BATSE instrument.
} 
Moreover, our analysis of the outlying SWIFT detection of GRB050525, whose time-lag we find to be $0.1261 \pm 0.0158 \mathrm{~s}$, is also in a good agreement with other calculations [42].

It is immediately apparent from Fig. 1 that there is a trend for $\Delta t$ to decrease as $K$ increases. Indeed, the linear fit shown in the left panel corresponds to

$$
\frac{\Delta t_{\mathrm{obs}}^{\mathrm{tot}}}{1+z}=(-0.172 \pm 0.021) K+(0.063 \pm 0.008)
$$

The unwary might conclude, prematurely, that there is a very significant Lorentz-violating effect, since the coefficient of the linear term in (6) is formally many standard deviations from zero.

However, as seen in the right panel of Fig. 1, the Lorentz-violating slope $a_{\mathrm{LV}}$ and the intrinsic time-lag $b_{\text {sf }}$ are very highly correlated. The appropriate tool for estimating the significance of any Lorentz-violating effect is the marginal distribution of the slope parameter in the fit (6), as obtained by integrating over the intercept parameter. Taking into account the correlation matrix as described in [47], we rescale by a factor $\sqrt{1-\rho^{2}}$ the Gaussian-like shape of this marginal distribution, where $\rho$ is the correlation coefficient of the bivariate slope-intercept distribution. The mean value is still unchanged at $a_{\mathrm{LV}, \text { obs }(\operatorname{marg})}^{\text {tot }}=$ -0.172 , whereas the variance (defined as the width at the half maximum) is $\sigma_{a_{\mathrm{LV}, \text { obs(marg) }}^{\text {tot }}}=$ 0.018. The mean value of the slope in this marginalized distribution is therefore negative even with a higher level of significance. However, the linear fit has a very large value of $\chi^{2} /$ d.o.f. $=149 / 33$, which indicates that either some errors are underestimated, which is often the case when data from different instruments are used in a common fit, or there is an additional uncertainty not yet taken into account.

The first possibility can be modelled by rescaling the errors in all the extracted time-lags by a scale factor $S=\left[\chi^{2} / \text { d.o.f. }\right]^{1 / 2}[49]$, guaranteeing that the resulting $\chi^{2} /$ d.o.f becomes unity. The corresponding linear fit has central values identical to those found with the original estimated errors, but the errors in both the tilt and the intercept are increased by a factor $\sqrt{S}$ :

$$
\left(\frac{\Delta t_{\mathrm{obs}}^{\mathrm{tot}}}{1+z}\right)_{\text {scale }}=(-0.172 \pm 0.045) K+(0.062 \pm 0.017)
$$

This procedure yields naively $3.8 \sigma$ evidence for a negative tilt in (7), which becomes $4.5 \sigma$ when one calculates the marginalized distribution for the slope parameter $a_{\mathrm{LV}}$. This would still suggest significant evidence for Lorentz violation, with the null hypothesis of Lorentz invariance having a probability of only $10^{-5}$.

However, any exceptional claim requires exceptional evidence, so we must scrutinize this 'signal' very carefully. Instead of rescaling all the errors uniformly, one might prefer to deweight the time-lags associated with some of the features whose identification is less reliable. In the absence of clear understanding of the GRB sources, we propose the following criterion for selecting a subsample of the time structures whose analysis may be less unreliable. Following [50], we assume that individual pulses are mostly related to single emission episodes. Therefore, we retain only those 'genuine' variation points which appear 

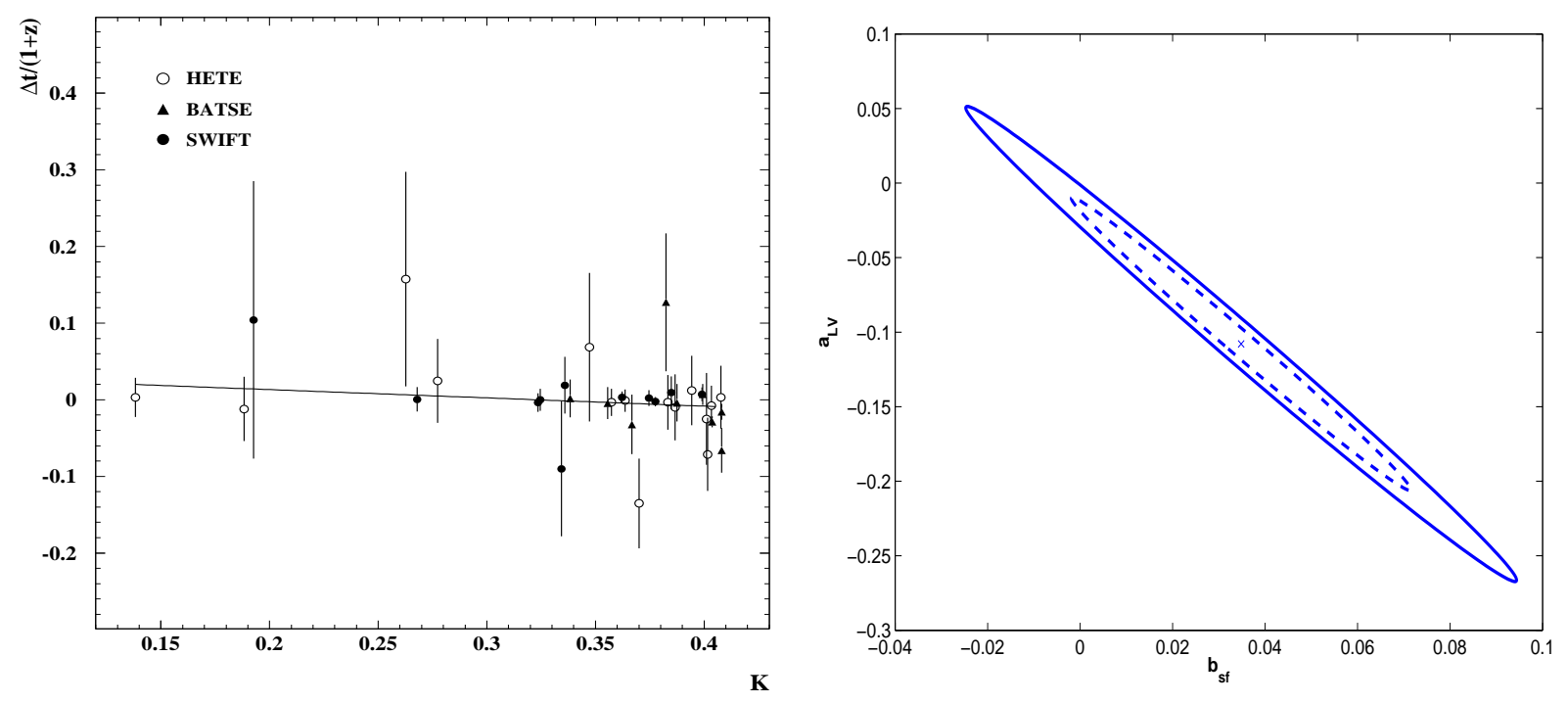

Figure 2: Left panel: Same as Fig. 1 but using only those high-intensity genuine variation points thought to be more reliable. Also, the errors have been rescaled by a universal factor $S$ according to the procedure [49] described in the text, so that $\chi^{2} /$ d.o.f. $=1$. Right panel: The error ellipse in the slope-intercept plane for the fit (8). The 68\% and 95\% confidencelevel contours are represented by the inner and outer lines, respectively.

in the vicinity of the highest pulse in a given light curve, whereas in the previous overall fit in the left panel of Fig. 1 we used the weighted averages of the differences between the positions of all the 'genuine' variation points and their counterparts in softer energy bands found in a given light curve. Specifically, we select only those variation points at which the amplitudes reach $70 \%$ of the maximal count rate for a given light curve. In addition to this choice, we further restrict our attention to the variation structure with the highest signalto-noise ratio in any given light curve. The resulting selection of time-lags is shown in the left panel of Fig 2, together with a linear fit to the de-weighted data. Although the data still exhibit significant scatter, the furthest outlying points have now disappeared. The corresponding slope and intercept parameters are given by the following fit, after rescaling by the appropriate $S$ factor:

$$
\left(\frac{\Delta t_{\mathrm{obs}}^{\mathrm{dw}}}{1+z}\right)_{\text {scale }}=(-0.108 \pm 0.065) K+(0.035 \pm 0.024) .
$$

This fit also indicates a negative value of the slope parameter. The strong correlation between the slope and intrinsic time-lag parameters persists, as seen in the right panel of Fig. 2, and the marginalized distribution for the slope yields a $1.9 \sigma$ effect.

Since the significance of the effect decreases substantially when we select a supposedly more reliable subsample, we conclude that there is no robust evidence for a vacuum refractive index.

The rescaling of the errors by a universal factor $S$ was motivated by our ignorance 

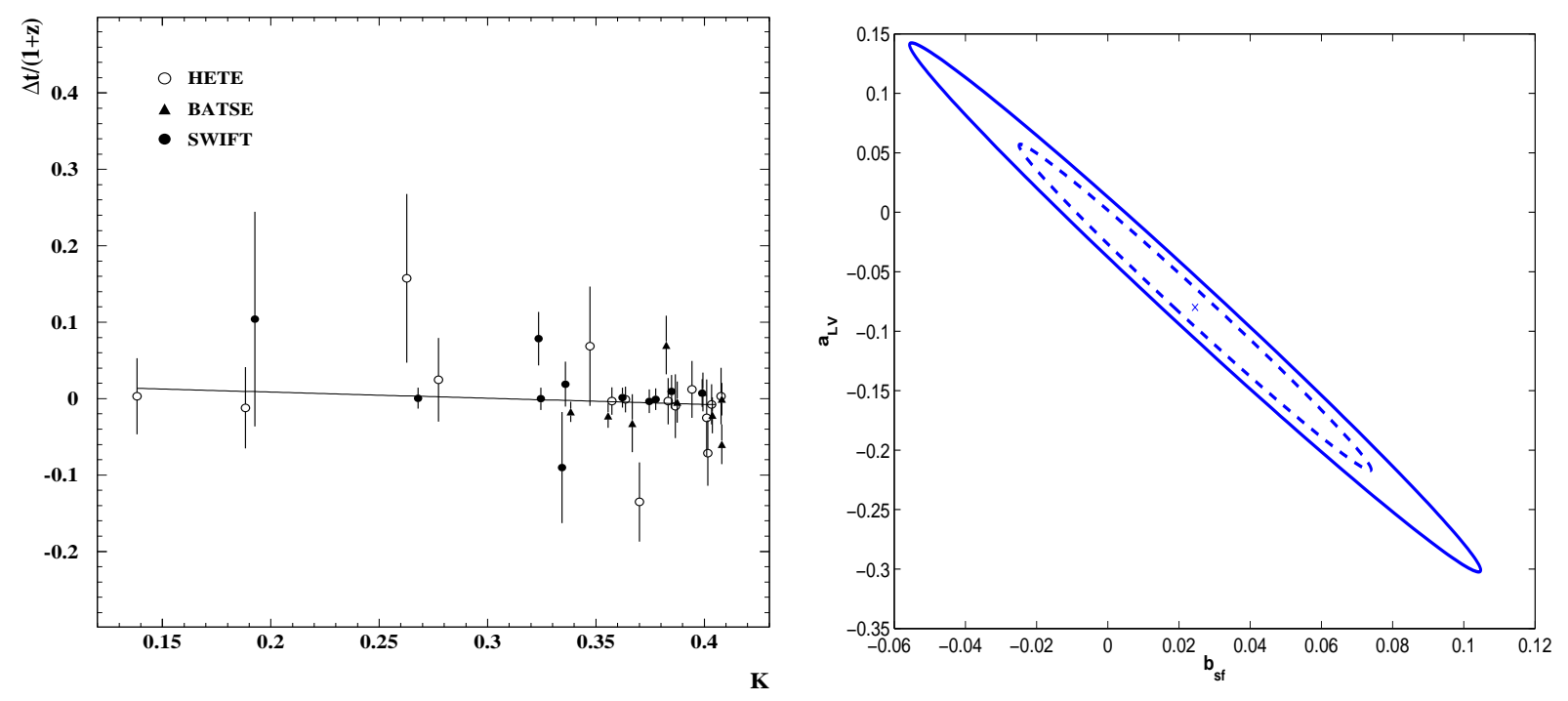

Figure 3: Left panel: Same as Fig. 1 but with the fitted errors in the intrinsic time-lags increased by $54 \mathrm{~ms}$, modelling a possible stochastic spread at the sources, as discussed in the text. The corresponding linear fit has a $\chi^{2} /$ d.o.f. of unity. Right panel: The error ellipse in the slope-intercept plane for the fit (9). The 68\% and $95 \%$ confidence-level contours are represented by the inner and outer lines, respectively.

of the origins of the systematic effects that are clearly present in our data set. However, since most of the GRBs exhibit more than one 'genuine' feature (in the Lipschitz/wavelet sense described above), which generally have different time-lags, a combined spectral lag can only be a crude measure of the time delays within a given burst. For example, since the burst evolves in time, its internal dynamics may cause different pulses within a burst to exhibit different intrinsic time-lags. Various analysis methods are available to examine these effects $[48,51,52,53]$, to which our wavelet approach [16] may be added. However, the degree of evolution of time-lags is yet to be well quantified, and there may be other environmental effects not directly related to the evolutionary stage.

One simplistic way to take such systematic uncertainties into account would be, instead of the previous universal rescaling of the errors, to allow for a universal stochastic spread in the intrinsic time-lag at the source. This may be done by adding in quadrature, for all the GRBs, a universal source error whose normalization is then fixed so that $\chi^{2} /$ d.o.f. $=1$. The corresponding universal source error is estimated in this way to be $54 \mathrm{~ms}$. A linearregression fit with this representation of stochastic intrinsic time-lags is presented in the left panel of Fig. 3, and leads to

$$
\left(\frac{\Delta t_{\mathrm{obs}}^{\mathrm{tot}}}{1+z}\right)_{\text {stochastic }}=(-0.080 \pm 0.091) K+(0.025 \pm 0.033) .
$$

This universal source error of $54 \mathrm{~ms}$ is well inside the resolution of all the instruments whose data we use. The fit (9) again yields naively a negative value of the slope parameter, but 
now only at the $0.9 \sigma$ level. However, the slope and intrinsic time-lag parameters are still highly correlated, as seen in the right panel of Fig. 3, and the significance becomes $1 \sigma$ when the marginalized distribution is computed. This certainly cannot be considered as evidence for a vacuum refractive index.

If we restrict our attention to the subset of 'more reliable' variation points described earlier, and allow for stochastic fluctuations in their intrinsic time-lags, we find that an error of $34 \mathrm{~ms}$ would give $\chi^{2} /$ d.o.f. of unity for this subset of variation points. This tends to confirm our suggestion that the selected points are indeed more reliable. After including this stochastic intrinsic time-lag, the linear regression fit for this 'reliable' set becomes

$$
\left(\frac{\Delta t_{\mathrm{obs}}^{\mathrm{dw}}}{1+z}\right)_{\text {stochastic }}=(-0.086 \pm 0.074) K+(0.028 \pm 0.027),
$$

which is rather similar to (9).

We therefore quote only a lower limit on the quantum-gravity scale parameter $M$. To determine this, we first find the regions of the slope-intercept plane that are allowed at various confidence levels. In the class of quantum-gravity models we wish to explore, the slope parameter must be positive semi-definite, as also suggested by considering the possibility of gravitational Čerenkov radiation [54]. Accordingly, we first quote a limit on the scale of violation of Lorentz invariance in the Bayesian manner proposed in [55], where the confidence range was constructed for a Gaussian distribution with negative mean which is constrained physically to be non-negative. We apply this Bayesian approach to the 'genuine' variation points, with errors rescaled universally: see (8) and Fig. 2. Using the prescription of [55] for the measured negative mean of the marginalized slope distribution at $2 \sigma$ below zero, we calculate the $95 \%$ confidence limit on the scale of violation of Lorentz invariance assuming a random variable obeying Gaussian statistics with a boundary at the origin. The corresponding upper limit on the positive value of the slope parameter is $a_{\mathrm{LV}}^{\min }=0.008$. Substituting $a_{\mathrm{LV}}^{\min }$ into the prefactor formula (1), together with the energy difference between the upper and lower BATSE spectral bands, we find the lower limit

$$
M \geq 1.8 \times 10^{16} \mathrm{GeV}
$$

from (8) and Fig. 2. This becomes

$$
M \geq 0.9 \times 10^{16} \mathrm{GeV}
$$

when we use (10). A similar result would be obtained from (9) and Fig. 3.

As an alternative to this Bayesian approach, we have also analyzed the likelihood function $L \propto \exp \left(-\chi^{2}(M) / 2\right)$, where we evaluated $\chi^{2}$ using

$$
\chi^{2}(M)=\sum_{\mathcal{D}}\left[\frac{\frac{\Delta t_{i}}{1+z_{i}}-a_{\mathrm{LV}}(M) K_{i}-b_{\mathrm{sf}}}{\frac{\sigma_{i}}{1+z_{i}}}\right]^{2} .
$$

The sum in (13) is taken over the all data points $\Delta t_{i}$, symbolized by $\mathcal{D}$, with $\sigma_{i}$ characterizing the uncertainties in the measured time-lags. We have calculated $L$ for all the 


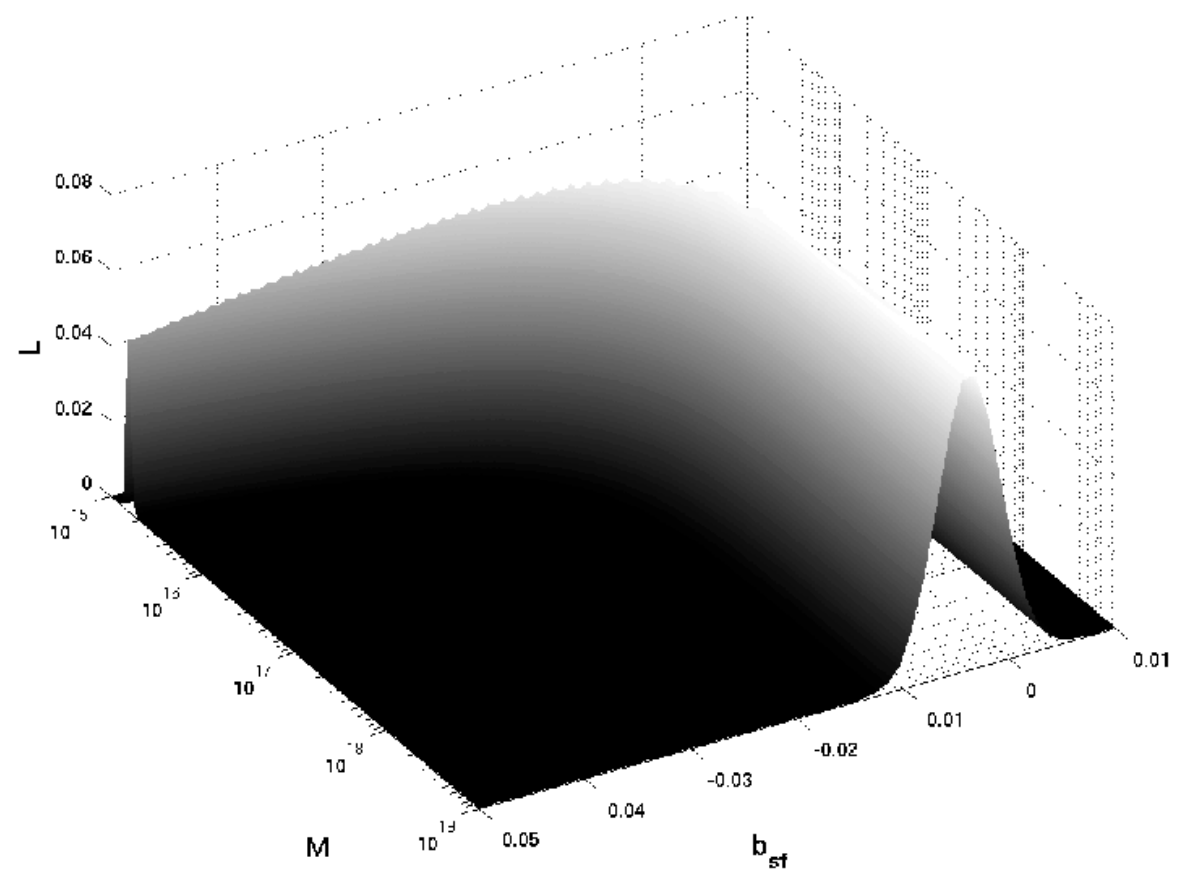

Figure 4: The likelihood surface in the parameter space of the intrinsic time-lag $b_{\mathrm{sf}}$ and the Lorentz violation scale $M$.

possible values of $a_{\mathrm{LV}}(M)$, as defined by the coefficient of $K$ in (1) and a reasonable range of the a priori unknown intercept parameter $b_{\mathrm{sf}}$. The likelihood surface is shown in Fig. 4 . Marginalizing this likelihood function over the intercept parameter $b_{\text {sf }}$, one can establish 95\% confidence limit on the scale $M$ of Lorentz violation by solving the equation

$$
\frac{\int_{M}^{M_{\infty}} L_{\operatorname{marg}}(\xi) d \xi}{\int_{0}^{M_{\infty}} L_{\operatorname{marg}}(\xi) d \xi}=0.95
$$

where $\infty$ symbolizes a reference point fixing the normalization. In our case, we choose as reference point $M_{\infty}=10^{19} \mathrm{GeV}$, the Planck mass. In practice varying this reference point by as much as an order of magnitude influences the final result only very weakly. The $95 \%$ confidence-level lower limit obtained in this way from the more reliable subset of variation points is

$$
M \geq 2.1 \times 10^{16} \mathrm{GeV}
$$

if we rescale the errors by a universal factor, and becomes $M \geq 1.6 \times 10^{16} \mathrm{GeV}$ if we allow for stochastic time-lags at the sources for the robust variation points. It is reassuring that the two ways of calculating the lower limit on the quantum-gravity scale yield similar numbers (12), (15). Conservatively, we prefer to quote the weaker lower limit (12) at the level of one significant figure. We have also explored the effect of dropping any one of the BATSE, SWIFT and HETE data sets, finding that the tendency of the slope parameter 
to be negative is always preserved, though the significance is (unsurprisingly) reduced somewhat in each case.

\section{Discussion}

The result of the present analysis is significantly stronger and more robust than that in [16], thanks to our improved statistical technique and the use of a more complete data set. The most conservative limit on the violation of Lorentz invariance that we find using the most luminous parts of the GRB emissions is $M \geq 0.9 \times 10^{16} \mathrm{GeV}$. Without this conservative approach, using the whole data set we would have found strong evidence in favour of Lorentz violation, with a probability of only $10^{-5}$ for Lorentz invariance.

It is instructive to compare this analysis with that in [16], where we found $M \geq$ $6.9 \times 10^{15} \mathrm{GeV}$. Here we benefit from using a larger sample of GRBs with known redshifts, some of which provide variation points whose time-lags have small errors, and the sensitivity of this analysis might have been expected to be an order of magnitude greater than in [16]. However, the strong correlation between the parameters describing intrinsic and propagation time-lags and the large value of the overall $\chi^{2} /$ d.o.f. have revealed new systematic issues. The sensitivity of the new data set to $M$ is reduced by the need either to rescale the errors [49] or to allow for stochastic intrinsic time-lags. Moreover, whereas previously we just used a likelihood analysis, which in this case would have yielded $M \geq 2.1 \times 10^{16} \mathrm{GeV}$ (a factor three better than in [16]), here we also use the Bayesian approach [55], which yields a somewhat weaker limit. In order to be conservative, this is the final limit we wish to quote.

Some stronger upper limits on a linear modification of the photon dispersion relation have been reported in the literature. These have also been based on the type of time-offlight analysis proposed in [8], but using individual sources: either a single GRB [10, 11] or a single AGN flare [9]. As our analysis shows, any analysis of the time of flight of radiation from a single source $[9,10,11]$ can only be regarded as indicative, since it is vulnerable to unknown systematic uncertainties associated with the unknown intrinsic spectral properties of any given transient source. In order to establish a rigorous limit, and even more to establish any non-zero effect, one must be able to distinguish between intrinsic and propagation effects, which can be done robustly only by analyzing a sizeable statistical sample of sources. In astronomical parlance, the quantum-gravity energy-dependent time delay described by (1) is the analogue of a foreground effect superimposed on the original spectral properties of the GRBs or other sources. The only way to minimize these unknown intrinsic systematic uncertainties is to search for a significant linear correlation of the measured time-lags with the redshifts. We have learned from our analysis that it is difficult to break the degeneracy between the slope (propagation) and intercept (source) parameters.

An analysis [11] of the individual source GRB021206 reported a stronger lower limit on the scale of violation of Lorentz invariance for photon propagation of $M \geq 1.8 \times 10^{17} \mathrm{GeV}$, using the relation (1). However, the redshift of GRB021206 was not measured directly, and instead the estimate $z=0.3-0.6$ used in [11] was taken from [56]. We made the 
exercise of including GRB021206 in our data fit, assuming a redshift at either end of this estimated range. If we assume $z=0.3$, we find $a_{\mathrm{LV}}=-0.039 \pm 0.016$, which corresponds to roughly the same limit as that we obtain. Assuming that this GRB has redshift $z=0.6$ would disfavour a positive slope at the level of $5 \sigma$, but this is unjustified in the absence of a measured error for the redshift of GRB021206. Moreover, assigning to the time-lag for GRB021206 the same stochastic source uncertainty of $54 \mathrm{~ms}$ that we found for our the other GRBs with known redshifts and combining it with our data set would give results very similar to those we quote for either $z=0.3$ or $z=0.6$.

Quite generally, one would need to demonstrate the absence of any destructive interference between intrinsic and propagation effects, which has not been done, and is unlikely to be possible for a single source. In our view, the only way to disentangle the properties of the source from propagation effects, and thereby minimize potential systematic errors, is to look for a correlation with redshift in a statistical sample of GRBs with a spread of different measured redshifts. On this basis, the available GRB data can be used conservatively to set a lower limit on any possible quantum gravity effect at the level (12). The systematic effects we have found at low and moderate redshifts cast severe doubts on the use of individual GRBs with unknown redshifts $[11,10]$ to claim a high sensitivity to violations of Lorentz invariance using such a of time-of-flight approach.

Similar arguments apply to time-of-flight measurements using $\mathrm{TeV} \gamma$-flares from blasars [9]. Moreover, the situation with systematic uncertainties in this case is even less under control than for GRBs, because so far only the single object Mrk421 has exhibited a short-duration flare, so there is no way to estimate the systematic uncertainties inherited from the source, as we have done here for GRBs using a regression analysis.

Unfortunately, significant progress beyond our limit (12) may not be possible until the internal dynamics of GRBs is better understood or data extending to much higher energies become available.

\section{Acknowledgments}

The work of D.V.N. was supported in part by DOE grant DE-FG03-95-ER-40917.

\section{References}

[1] S. Sarkar, Mod. Phys. Lett. A 17 (2002) 1025 [arXiv:gr-qc/0204092]; E. Gravanis and N. E. Mavromatos, arXiv:hep-th/0108008; L. Smolin, arXiv:hep-th/0303185; Y. J. Ng, Mod. Phys. Lett. A 18 (2003) 1073 [arXiv:gr-qc/0305019]; T. Piran, arXiv:astroph/0407462; D. Mattingly, arXiv:gr-qc/0502097;

[2] G. Amelino-Camelia, J. R. Ellis, N. E. Mavromatos and D. V. Nanopoulos, Int. J. Mod. Phys. A 12 (1997) 607 [arXiv:hep-th/9605211]. 
[3] J. R. Ellis, N. E. Mavromatos and D. V. Nanopoulos, Phys. Lett. B 293 (1992) 37 [arXiv:hep-th/9207103]; For reviews see: J. R. Ellis, N. E. Mavromatos and D. V. Nanopoulos, Erice Subnucl. Phys. Series, Vol. 311 (World Sci. 1994) [arXiv:hepth/9311148]; J. Chaos, Solitons and Fractals, Vol. 10 (1999) 345 (eds. C. Castro and M.S. El Naschie, Elsevier Science, Pergamon 1999) [arXiv:hep-th/9805120].

[4] R. Gambini and J. Pullin, Phys. Rev. D 59 (1999) 124021 [arXiv:gr-qc/9809038]; J. Alfaro, H. A. Morales-Tecotl and L. F. Urrutia, Phys. Rev. D 65 (2002) 103509 [arXiv:hep-th/0108061].

[5] V. A. Kostelecky and S. Samuel, Phys. Rev. D 39 (1989) 683.

[6] G. Amelino-Camelia, Int. J. Mod. Phys. D 11 (2002) 35 [arXiv:gr-qc/0012051].

[7] R. C. Myers and M. Pospelov, Phys. Rev. Lett. 90 (2003) 211601 [arXiv:hep$\mathrm{ph} / 0301124]$.

[8] G. Amelino-Camelia, J. Ellis, N. Mavromatos, D. Nanopoulos and S. Sarkar, Nature 393 (1998) 763.

[9] S.D. Biller et al., Phys. Rev. Lett. 83 (1999) 2108.

[10] B.E. Schafer, Phys. Rev. Lett. 82 (1999) 4964.

[11] S. E. Boggs, C. B. Wunderer, K. Hurley and W. Coburn, Astrophys. J. 611 (2004) L77 [arXiv:astro-ph/0310307].

[12] P. Kaaret, arXiv:astro-ph/9903464.

[13] T. Jacobson, S. Liberati and D. Mattingly, Phys. Rev. D 66 (2002) 081302. [arXiv:hep$\mathrm{ph} / 0112207]$.

[14] J. Alfaro and G. Palma, Phys. Rev. D 67 (2003) 083003 [arXiv:hep-th/0208193]; F. W. Stecker and S. L. Glashow, Astropart. Phys. 16 (2001) 97 [arXiv:astroph/0102226]; S. R. Coleman and S. L. Glashow, Phys. Rev. D 59 (1999) 116008 [arXiv:hep-ph/9812418]; R. J. Protheroe and H. Meyer, Phys. Lett. B 493 (2000) 1 [arXiv:astro-ph/0005349]; G. Amelino-Camelia and T. Piran, Phys. Rev. D 64 (2001) 036005 [arXiv:astro-ph/0008107]; G. Amelino-Camelia, New J. Phys. 6, 188 (2004) [arXiv:gr-qc/0212002]; T. J. Konopka and S. A. Major, New J. Phys. 4 (2002) 57 [arXiv:hep-ph/0201184]; F. W. Stecker, New Astron. Rev. 48 (2004) 437 [arXiv:astroph/0304527]; F. W. Stecker, Astropart. Phys. 20 (2003) 85 [arXiv:astro-ph/0308214];

[15] V. A. Kostelecky and M. Mewes, Phys. Rev. D 66 (2002) 056005 [arXiv:hep$\mathrm{ph} / 0205211]$.

[16] J. R. Ellis, N. E. Mavromatos, D. V. Nanopoulos and A. S. Sakharov, Astron. Astrophys. 402 (2003) 409 [arXiv:astro-ph/0210124]. 
[17] BATSE data archive:

ftp://legacy.gsfc.nasa.gov/compton/data/batse/ascii_data/64ms/,

[18] HETE data archive: http://space.mit.edu/HETE/Bursts/

[19] SWIFT data archive: http://swift.gsfc.nasa.gov/docs/swift/archive/.

[20] J. R. Ellis, N. E. Mavromatos and D. V. Nanopoulos, arXiv:gr-qc/9909085.

[21] N. A. Bahcall, J. P. Ostriker, S. Perlmutter and P. J. Steinhardt, Science 284 (1999) 1481 [astro-ph/9906463].

[22] J. Ellis, K. Farakos, N.E. Mavromatos, V.A. Mitsou and D.V. Nanopoulos, Ap. J. 535 (2000) 139.

[23] Compilation by J. Greiner:

http://www .mpe.mpg.de/ jcg/grbgen.html.

[24] M. R. Metzger et al., Nature 387 (1997) 878.

[25] S. R. Kulkarni et al., Nature 395 (1998) 663.

[26] S. G. Djorgovski et al., Astrophys. J. 508 (1998) L17. arXiv:astro-ph/9808188.

[27] S. R. Kulkarni et al., Nature 398 (1999) 389.

[28] B.E. Schafer et al., Astrophys. J. 524 (1999) L103.

[29] K. Buermann et al., Astron. Astrophys. 352 (1999) L26.

[30] P. M. Vreeswijk et al., GCN 496 (1999); L. Piro et al., of Science 290 (2000) 955 [arXiv:astro-ph/0011337].

[31] J. S. Bloom et al., Astrophys. J. 125 (2003) 999.

[32] P. A. Price et al., AIP Conf. Proc. 662 (2003) 56 [arXiv:astro-ph/0201399].

[33] J. Hjorth et al., Astrophys. J. 597 (2003) 699 [arXiv:astro-ph/0307331].

[34] A. M. Soderberg et al., Astrophys. J. 606 (2004) 994 [arXiv:astro-ph/0311050].

[35] A. J. Barth et al., Astrophys. J. 584 (2003) L47 [arXiv:astro-ph/0212554].

[36] P. Jakobsson et al., Astrophys. J. 629 (2005) 45 [arXiv:astro-ph/0505035].

[37] P. Moller et al., Astron. Astrophys. 396 (2002) L21 [arXiv:astro-ph/0210654].

[38] P. M. Vreeswijk et al., Astron. Astrophys. 419 (2004) 927 [arXiv:astro-ph/0403080].

[39] K. Z. Stanek et al., Astrophys. J. 591 (2003) L17 [arXiv:astro-ph/0304173]. 
[40] P. Jakobsson et al., Astron. Astrophys. 427 (2004) 785 [arXiv:astro-ph/0407439].

[41] P. R. Wozniak, W. T. Vestrand, J. A. Wren, R. R. White, S. M. Evans and D. Casperson, Astrophys. J. 627 (2005) L13 [arXiv:astro-ph/0505336].

[42] A. J. Blustin et al. [Swift Collaboration], arXiv:astro-ph/0507515.

[43] E. Berger et al., arXiv:astro-ph/0508115.

[44] R. L. C. Starling et al., arXiv:astro-ph/0508237.

[45] G. Cusumano et al., arXiv:astro-ph/0509737; G. Tagliaferri et al., arXiv:astro$\mathrm{ph} / 0509766$.

[46] D.L. Donoho, Proc. Symp. Appl. Math. 47 (Ed. I. Daubechies), 173 (1993); D.L. Donoho, I.M. Jonston, J. Kerkyacharian and D. Picard, J. R. Stat. Soc. B57, 301 (1995).

[47] R. J. Barlow A Guide to the Use of Statistical Methods in the Physical Sciences (The Manchester Physical Series, 1999).

[48] J.P. Norris, G.F. Marani, J.T. Bonnell, Ap. J. 534 (2000) 248.

[49] S. Eidelman et al., Particle Data Group, Phys. Lett. B 592 (2004) 1.

[50] T. Piran, Phys. Rep. 314 (1999) 575 [arXiv:astro-ph/9810256].

[51] J. P. Norris, Astrophys. J. 579 (2002) 386.

[52] F. Ryde, arXiv:astro-ph/0411206.

[53] F. Ryde, D. Kocevski, Z. Bagoly, N. Ryde and A. Meszaros, arXiv:astro-ph/0411219.

[54] G. D. Moore and A. E. Nelson, JHEP 0109 (2001) 023 [arXiv:hep-ph/0106220].

[55] G. J. Feldman and R. D. Cousins, Phys. Rev. D 57 (1998) 3873 [arXiv:physics/9711021].

[56] J. L. Atteia, Astron. Astrophys. 407 (2003) L1 [arXiv:astro-ph/0304327]. 


\begin{tabular}{|cccc|}
\hline GRB & $z$ & $z$ Refs. & $\Delta t_{\text {total }}^{\left(\mathrm{E}_{\text {high }}-\mathrm{E}_{\text {low }}\right)}(\mathrm{s})$ \\
\hline \hline \multicolumn{3}{c}{ BATSE $(64 \mathrm{~ms})$} \\
\hline 970508 & 0.835 & {$[24]$} & $-0.059 \pm 0.044$ \\
\hline 971214 & 3.418 & {$[25]$} & $-0.098 \pm 0.045$ \\
\hline 980329 & 3.9 & {$[23]$} & $-0.084 \pm 0.036$ \\
\hline 980703 & 0.966 & {$[26]$} & $0.138 \pm 0.053$ \\
\hline 990123 & 1.600 & {$[27]$} & $-0.155 \pm 0.041$ \\
\hline 990308 & 1.2 & {$[28]$} & $0.0188 \pm 0.0138$ \\
\hline 990510 & 1.619 & {$[29]$} & $-0.0017 \pm 0.0143$ \\
\hline 991216 & 1.020 & {$[30]$} & $-0.0091 \pm 0.0012$ \\
\hline 990506 & 1.3060 & {$[31]$} & $-0.0503 \pm 0.0075$ \\
\hline & & HETE $(164 \mathrm{~ms})$ & \\
\hline 010921 & 0.45 & {$[32]$} & $0.0357 \pm 0.0585$ \\
\hline 020124 & 3.198 & {$[33]$} & $-0.0046 \pm 0.0455$ \\
\hline 020903 & 0.25 & {$[34]$} & $-0.0150 \pm 0.0386$ \\
\hline 020813 & 1.25 & {$[35]$} & $-0.1602 \pm 0.0794$ \\
\hline 020819 & 0.41 & {$[36]$} & $0.222 \pm 0.145$ \\
\hline 021004 & 2.33 & {$[37]$} & $-0.0402 \pm 0.1109$ \\
\hline 021211 & 1.01 & {$[23]$} & $-0.0202 \pm 0.0639$ \\
\hline 030226 & 1.99 & {$[23]$} & $-0.0227 \pm 0.0568$ \\
\hline 030323 & 3.372 & {$[38]$} & $-0.0148 \pm 0.0570$ \\
\hline 030328 & 1.52 & {$[23]$} & $0.00825 \pm 0.07661$ \\
\hline 030329 & 0.168 & {$[39,23]$} & $0.0037 \pm 0.0219$ \\
\hline 030429 & 2.66 & {$[40]$} & $-0.0123 \pm 0.0965$ \\
\hline 040924 & 0.859 & {$[23]$} & $-0.2516 \pm 0.0801$ \\
\hline 041006 & 0.716 & {$[23]$} & $0.1179 \pm 0.1228$ \\
\hline 050408 & 1.2357 & {$[23]$} & $-0.0562 \pm 0.0989$ \\
\hline & & $\mathrm{SWIFT}(64 \mathrm{~ms})$ & $0.0054 \pm 0.0109$ \\
\hline 050319 & 3.24 & {$[41]$} & $-0.0135 \pm 0.0285$ \\
\hline 050401 & 2.9 & {$[23]$} & $-0.1491 \pm 0.1075$ \\
\hline 050416 & 0.653 & {$[23]$} & $-0.0012 \pm 0.0561$ \\
\hline 050505 & 4.3 & {$[23]$} & $0.1261 \pm 0.0159$ \\
\hline 050525 & 0.606 & {$[23,42]$} & $-0.0032 \pm 0.0047$ \\
\hline 050603 & 2.821 & {$[23]$} & $0.131 \pm 0.1681$ \\
\hline 050724 & 0.258 & {$[43]$} & $0.094 \pm 0.1361$ \\
\hline 050730 & 3.968 & {$[44]$} & $0.033 \pm 0.0569$ \\
\hline 050820 & 2.612 & {$[23]$} & $0.004 \pm 0.0852$ \\
\hline 050904 & 6.29 & {$[45]$} & \\
\hline 050922 & 2.17 & {$[23]$} & \\
\hline & & & \\
\hline
\end{tabular}

Table 1: Data on spectral time-lags for GRBs with known redshifts collected by the BATSE, HETE and SWIFT instruments. 